\title{
Some Refinements and Generalizations of I. Schur Type Inequalities
}

\author{
Xian-Ming Gu, ${ }^{1}$ Ting-Zhu Huang, ${ }^{1}$ Wei-Ru Xu, ${ }^{2}$ Hou-Biao Li, ${ }^{1}$ Liang Li, ${ }^{1}$ and Xi-Le Zhao \\ ${ }^{1}$ School of Mathematical Sciences, University of Electronic Science and Technology of China, Chengdu 611731, China \\ ${ }^{2}$ School of Science, North University of China, Taiyuan 030051, China \\ Correspondence should be addressed to Ting-Zhu Huang; tingzhuhuang@126.com
}

Received 18 December 2013; Accepted 6 February 2014; Published 16 March 2014

Academic Editors: L. Acedo, Y.-M. Chu, and B.-N. Guo

Copyright (c) 2014 Xian-Ming Gu et al. This is an open access article distributed under the Creative Commons Attribution License, which permits unrestricted use, distribution, and reproduction in any medium, provided the original work is properly cited.

Recently, extensive researches on estimating the value of $e$ have been studied. In this paper, the structural characteristics of I. Schur type inequalities are exploited to generalize the corresponding inequalities by variable parameter techniques. Some novel upper and lower bounds for the I. Schur inequality have also been obtained and the upper bounds may be obtained with the help of Maple and automated proving package (Bottema). Numerical examples are employed to demonstrate the reliability of the approximation of these new upper and lower bounds, which improve some known results in the recent literature.

\section{Introduction}

It is well known that $x_{n}=(1+(1 / n))^{n}$ and $y_{n}=(1+$ $(1 / n))^{n+1}$ are, respectively, monotone increasing and monotone decreasing, and both of them converge to the constant $e$. In fact, extensive researches for the estimated value of $e$ have been studied [1-4], and the methods for estimating the value of $e$ are of benefit to the improvements of the Hardy inequality, Carleman inequality, Gamma function inequality, and so forth [5-13], which is an essential motivation for this work. Klambauer and Schur have reached the following conclusion.

Lemma 1 (see [14]). Both $s_{n}=(1+(1 / n))^{n+\alpha}$ and $t_{n}=(1+$ $(1 / n))^{n+1}(1+(\alpha / n))$ are monotone decreasing sequences if and only if $\alpha \geq(1 / 2)$.

In fact, it is not hard to prove that

$$
e<\left(1+\frac{1}{n}\right)^{n+(1 / 2)}=\sqrt{1+\frac{1}{n}}\left(1+\frac{1}{n}\right)^{n}
$$

which has been proved by different ways; refer to [14-17].
Besides, Fischer and Qi had further studied this issue (see [18-20]) and they demonstrated that $x_{n}$ is a monotone increasing sequence if and only if

$$
\alpha \leq \frac{2 \ln 3-3 \ln 2}{2 \ln 2-\ln 3}=0.409 \ldots
$$

Moreover, Alzer and Qi have obtained the necessary and sufficient conditions for the monotonicity of generalized types of $b_{n}=(1+(\alpha / n))^{n+\beta}$ and $F_{\alpha, \beta}(x)=(1+(\alpha / x))^{x+\beta}$; see $[21,22]$ for details.

Recently, I. Schur has obtained the so-called I. Schur inequality as follows:

$$
p_{n}<e<q_{n}
$$

where $p_{n}=x_{n}(1+(1 /(2 n+1)))$ and $q_{n}=x_{n}(1+(1 / 2 n))$.

It can also solve the problem proposed by Klambauer in [15]: "Is the $e$ contained in a quarter of the interval of $(1+$ $(1 / n))^{n}<e<(1+(1 / n))^{n+1}$ ?" Therefore, here $e$ is included in the interval length of

$$
E=\left(1+\frac{1}{2 n}\right)-\left(1+\frac{1}{2 n+1}\right)=\frac{1}{2 n(2 n+1)} \leq \frac{1}{6},
$$


In [23], Xu and Yang have obtained a series of improved forms of the I. Schur inequalities, from which there is one conclusion which can be drawn that the necessary and sufficient condition for $(1+(1 / n))^{n}(1+(1 / a n))<e<(1+$ $(1 / n))^{n}(1+(1 / 2 n))$ is $a>(2 /(e-2))$.

Many new estimated values on $e$ have been obtained when researchers study the improvements of the Carleman inequality; see [24-26] for details. We mainly analyze the structural characteristics of I. Schur type inequalities and find out their upper and lower bounds are closely related to the mean value sequences of $x_{n}$ and $y_{n}$. At last, we generalized a series of conclusions on the developments of I. Schur inequality via introducing variable parameters.

The rest of work is organized as follows. In Section 2, upper and lower bounds for a series of the I. Schur type inequalities are improved. Meanwhile, novel I. Schur-like inequalities are obtained by means of the variable parameter method. Some numerical examples are given to show the reliability of the approximation of new upper and lower bounds in Section 3. Finally, the paper closes with conclusions in Section 4.

\section{Main Results and Proofs}

2.1. Improvements Based on Computation of Mean Value. In this section, we consider the arithmetic mean value $A_{n}$, geometric mean value $G_{n}$, logarithmic mean value $L_{n}$, harmonic mean value $H_{n}$, and antilogarithmic mean $\Omega_{n}$ of $x_{n}$ and $y_{n}$, respectively; they are

$$
\begin{aligned}
A_{n} & =\frac{x_{n}+y_{n}}{2}=\frac{(1+(1 / n))^{n}+(1+(1 / n))^{n+1}}{2} \\
& =x_{n}\left(1+\frac{1}{2 n}\right) \\
G_{n} & =\left(x_{n} y_{n}\right)^{1 / 2}=\left[\left(1+\frac{1}{n}\right)^{n}\left(1+\frac{1}{n}\right)^{n+1}\right]^{1 / 2} \\
& =x_{n}\left(1+\frac{1}{n}\right)^{1 / 2}, \\
H_{n} & =\frac{2 x_{n} y_{n}}{x_{n}+y_{n}}=x_{n}\left(1+\frac{1}{2 n+1}\right), \\
L_{n} & =\frac{y_{n}-x_{n}}{\ln y_{n}-\ln x_{n}} \\
& =\left[\frac{(1+(1 / n))^{n+1}-(1+(1 / n))^{n}}{\ln (1+(1 / n))^{n+1}-\ln (1+(1 / n))^{n}}\right] \\
& =\frac{x_{n}}{n \ln (1+(1 / n))}, \\
\Omega_{n} & =\frac{x_{n} y_{n}\left(\ln y_{n}-\ln x_{n}\right)}{y_{n}-x_{n}}=x_{n}\left(1+\frac{1}{n}\right) \ln x_{n} \\
& x_{n}(n+1) \ln \left(1+\frac{1}{n}\right),
\end{aligned}
$$

where $H_{n}$ is a monotone increasing sequence, see [14] for a discussion of this issue, which means that the left-hand side of I. Schur inequality is valid. A lower bound for I. Schur inequality can be obtained via the monotone convergence theorem [27, pp. 87-88]. Moreover, it has been confirmed that $A_{n}$ and $\Omega_{n}$ are monotone decreasing in [28]: both $A_{n}=$ $x_{n}(1+(1 / 2 n))$ and $\Omega_{n}=x_{n}(n+1) \ln (1+(1 / n))$ are monotone decreasing sequences, and we obtained two inequalities as follows:

$$
\begin{array}{r}
e<x_{n}\left(1+\frac{1}{2 n}\right), \quad e<x_{n}(n+1) \ln \left(1+\frac{1}{n}\right), \\
n=1,2, \ldots .
\end{array}
$$

The first inequality has verified the rationality of the righthand side of I. Schur inequality, and the second inequality has put forward a method for sharpening the upper bound of I. Schur inequality because of $(1+(1 / 2 n))>(n+1) \ln (1+$ $(1 / n)), n \in N^{*}$. Besides, the monotonicity of the remaining mean value sequences $G_{n}, L_{n}$ should also be checked, and the conclusions can be drawn as follows.

Proposition 2. $G_{n}=x_{n}(1+(1 / n))^{1 / 2}\left(n \in N^{*}\right)$ is a monotone decreasing sequence.

Remark 3. (1) We can also continue to construct geometric mean value sequence $Q_{n}=\left((1+(1 / n))^{2 n}(1+(1 / n))^{1 / 2}\right)^{1 / 2}=$ $x_{n}(1+(1 / n))^{1 / 4}$. By Lemma 1 and $(1 / 4)=0.25<((2 \ln 3-$ $3 \ln 2) /(2 \ln 2-\ln 3))=0.409 \ldots$, we can obtain that $Q_{n}=$ $x_{n}(1+(1 / n))^{1 / 4}$ is a monotone decreasing sequence; thus $x_{n}(1+(1 / n))^{1 / 4}<e$.

(2) Consider $m \geq 2, m \in N^{*}$, times of geometric mean value operation by combining with Lemma 1 and $\left(1 / 2^{m}\right)<$ $((2 \ln 3-3 \ln 2) /(2 \ln 2-\ln 3))=0.409 \ldots$, and we have $Q_{n}=x_{n}(1+(1 / n))^{1 / 2^{m}}<e$, which is a monotone increasing sequence for each $m$.

Theorem 4. $L_{n}=x_{n} /(n \ln (1+(1 / n)))$ is a monotone increasing sequence, and the following inequality

$$
L_{n}=\frac{x_{n}}{n \ln (1+(1 / n))}>e
$$

holds for $n \in N^{*}$.

Proof. We consider the monotonicity of $f(x)=(x / \ln x)$, $(1<x<e)$, and

$$
f^{\prime}(x)=\frac{\ln x-1}{(\ln x)^{2}},
$$

when $x \in(1, e), f^{\prime}(x)<0$ and hence $f(x)$ is monotone decreasing. It is known that $x_{n}=(1+(1 / n))^{n}$ is monotone increasing, and $x_{n}=(1+(1 / n))^{n} \in(1, e)$. According to monotonous property of the composite functions, $L_{n}=$ $x_{n} /(n \ln (1+(1 / n)))$ is a monotone decreasing sequence; thus

$$
f(x)=\frac{x}{\ln x}>f(e)=\frac{e}{\ln e}=e .
$$


Take $x=(1+(1 / n))^{n} \in(1, e)$; then

$$
L_{n}=\frac{x_{n}}{n \ln (1+(1 / n))}>e
$$

Based on this conclusion, it is not hard to note that it is an improvement of upper bound for I. Schur inequality. There is another conclusion shown as follows (see [14]).

Proposition 5. The double inequality

$$
\left(1+\frac{1}{n}\right)^{n+\alpha} \leq e \leq\left(1+\frac{1}{n}\right)^{n+\beta}
$$

for $n \geq 1$ is valid in the sense that the maximum $\alpha=(1 / \ln 2)-$ 1 and minimum $\beta=1 / 2$ in (11) are best possible.

Then we study the necessary and sufficient condition for the validness of the class of $(1+(1 /(2 n+\beta))) x_{n} \leq e$ and obtain the following.

Proposition 6. The inequality $(1+(1 /(2 n+\beta))) x_{n} \leq e$ holds if and only if $\beta \geq(5 / 6)$, and $\beta=5 / 6$ is the best constant.

In fact, the proof of this theorem can be introduced via the conclusions in [14] as follows:

$$
\frac{6 e}{12 x+11}<e-\left(1+\frac{1}{x}\right)^{x}<\frac{7 e}{14 x+12} \quad(x \geq 1) .
$$

Use the left-hand side of (12) and change $x$ into $n, n \in N^{*}$; then

$$
\left(1+\frac{1}{n}\right)^{n}<e-\frac{6 e}{12 n+11}=\frac{12 n+5}{12 n+11} e, \quad n \in N^{*}
$$

and we can also obtain $(1+(1 /(2 n+(5 / 6)))) x_{n} \leq e$. The conclusion on the best optimality of $\beta$ can be found in [13]. It needs to be mentioned that the authors have made a mistake when they cited this inequality in [14]; for instance, let $x=$ $(1 / 10)$; then $e-(1+(1 / x))^{x}=1.447300213 \ldots$, but $(7 e /(14 x+$ $12))=1.419997970 \ldots$. It means that the right-hand side of the inequality may not be valid. It is found that they mistake $x \geq 1$ for $x>0$ after checking the original paper [8]. In fact, we can prove $e-(1+(1 / x))^{x}<(e /(2 x+\alpha)), x>0$, and the constant $\alpha=e /(e-1)$ is the best possible.

Theorem 7. The sequence

$$
S_{n}=\frac{p_{n}+q_{n}}{2}=x_{n} \frac{8 n^{2}+8 n+1}{8 n^{2}+4 n}
$$

is monotone decreasing, and the inequality $x_{n}(1+(4 n+$ 1) $\left./\left(8 n^{2}+4 n\right)\right)>$ e holds for $n \in N^{*}$.
Proof. We consider $g(x)=\ln \left(8 x^{2}+8 x+1\right)-\ln \left(8 x^{2}+4 x\right)+$ $x \ln (x+1)-x \ln x, x>0$; then

$$
\begin{aligned}
g^{\prime}(x)= & \frac{16 x+8}{8 x^{2}+8 x+1}-\frac{16 x+4}{8 x^{2}+4 x} \\
& +\ln (x+1)-\ln x-\frac{1}{x+1}, \\
g^{\prime \prime}(x)= & \frac{16}{8 x^{2}+8 x+1}-\frac{(16 x+8)^{2}}{\left(8 x^{2}+8 x+1\right)^{2}} \\
& -\frac{16}{8 x^{2}+4 x}+\frac{(16 x+4)^{2}}{\left(8 x^{2}+4 x\right)^{2}}-\frac{1}{x(x+1)^{2}} \\
= & \left(128 x^{6}+384 x^{5}+488 x^{4}+336 x^{3}\right. \\
& \left.+125 x^{2}+21 x+1\right) \\
& \times\left(\left(8 x^{2}+8 x+1\right)^{2}(2 x+1)^{2}(x+1)^{2} x^{2}\right)^{-1}>0,
\end{aligned}
$$

where $x>0$. By $\lim _{x \rightarrow \infty} g^{\prime}(x)=0$, we obtain $g^{\prime}(x)<0$ $(x>0)$. Therefore, $S_{n}$ is monotone decreasing, and we can straightforwardly prove the desired inequality of the theorem with $\lim _{n \rightarrow \infty} S_{n}=e$.

Theorem 8. The sequence

$$
T_{n}=\frac{2 p_{n} q_{n}}{p_{n}+q_{n}}=x_{n} \frac{8 n^{2}+12 n+4}{8 n^{2}+8 n+1}
$$

is monotone decreasing, and the following inequality

$$
x_{n} \frac{8 n^{2}+12 n+4}{8 n^{2}+8 n+1}=x_{n}\left(1+\frac{4 n+3}{8 n^{2}+8 n+1}\right)>e
$$

holds for $n \in N^{*}$.

Proof. We consider $h(x)=\ln \left(8 x^{2}+12 x+4\right)-\ln \left(8 x^{2}+8 x+1\right)+$ $x \ln (x+1)-x \ln x, x \geq 1$, then $h^{\prime}(x)=\left((16 x+12) /\left(8 x^{2}+12 x+\right.\right.$ $4))-\left((16 x+8) /\left(8 x^{2}+8 x+1\right)\right)+\ln (x+1)+(x /(x+1))-\ln x-1$, and

$$
\begin{aligned}
h^{\prime \prime}(x)= & \frac{16}{8 x^{2}+12 x+4}-\frac{(16 x+12)^{2}}{\left(8 x^{2}+12 x+4\right)^{2}}-\frac{16}{8 x^{2}+8 x+1} \\
& +\frac{(16 x+8)^{2}}{\left(8 x^{2}+8 x+1\right)^{2}}+\frac{2}{x+1}-\frac{x}{(x+1)^{2}}-\frac{1}{x} \\
= & \frac{128 x^{4}+256 x^{3}+152 x^{2}+24 x-1}{x(x+1)\left(8 x^{2}+8 x+1\right)^{2}(2 x+1)^{2}}>0,
\end{aligned}
$$

where $x \geq 1$. According to $\lim _{x \rightarrow \infty} h^{\prime}(x)=0$, we obtain $h^{\prime}(x)<0(x \geq 1)$. Therefore, $T_{n}$ is monotone decreasing, and we can straightforwardly prove the desired inequality with $\lim _{n \rightarrow \infty} T_{n}=e$. 
In fact, the previous theorems can be viewed as the different improvements of original I. Schur inequality. The above improved inequalities follow the motivation of researching mean value sequences of the upper and lower bounds for original inequalities; the authors study the monotonicity of the sequences constructed by the mean value of upper and lower bounds for I. Schur inequality and its relationship with $e$. The arithmetic mean value should be replaced with the "weighted" mean value $W_{n}=\left(x_{n}+\lambda y_{n}\right) /(1+\lambda)=[1+\lambda /(\lambda+$ 1) $n] x_{n}, \lambda \in \mathbb{R}$; then we have the following.

Theorem 9. For $\lambda \in[(-1-\sqrt{3}) / 4,(-1+\sqrt{3}) / 4]$ and $n \in N^{*}$, the sequence $W_{n}$ is monotone decreasing, and the inequality $x_{n}[1+\lambda /(\lambda+1) n]<e$ holds.

Proof. We consider $d(x)=\ln (x+\lambda x+\lambda)-\ln (x+\lambda x)+x \ln (x+$ $1)-x \ln x(x>0)$; then

$$
\begin{aligned}
d^{\prime}(x)= & \frac{1+\lambda}{x+\lambda x+\lambda}-\frac{1+\lambda}{x+\lambda x}+\ln (x+1) \\
& +\frac{x}{x+1}-\ln x-1 \\
d^{\prime \prime}(x)= & -\frac{(1+\lambda)^{2}}{(x+\lambda x+\lambda)^{2}}+\frac{(1+\lambda)^{2}}{(x+\lambda x)^{2}}+\frac{2}{x+1} \\
& -\frac{x}{(x+1)^{2}}-\frac{1}{x} \\
= & \left(2 \lambda x^{2}+3 \lambda^{2} x^{2}+3 \lambda^{2} x\right. \\
& \left.+x^{3} \lambda^{2}-x^{3}+\lambda^{2}+2 \lambda x\right) \\
& \times\left((x+1)^{2} x^{2}(x+\lambda x+\lambda)^{2}\right)^{-1} .
\end{aligned}
$$

Now rewrite numerator of $(20)$ and denote $I_{x}(\lambda)=\left(3 x^{2}+\right.$ $\left.3 x+x^{3}+1\right) \lambda^{2}+\left(2 x+2 x^{2}\right) \lambda-x^{3}$. This is a quadratic function of $\lambda$, and its discriminant is

$$
\Delta=\left(2 x+2 x^{2}\right)^{2}+4\left(3 x^{2}+3 x+x^{3}+1\right) x^{3}>0
$$

We note that two roots of $I_{x}(\lambda)$ are

$$
\begin{aligned}
& \lambda_{1}=-\frac{\left(1+\sqrt{1+x^{2}+x}\right) x}{(x+1)^{2}}, \\
& \lambda_{2}=\frac{\left(-1+\sqrt{1+x^{2}+x}\right) x}{(x+1)^{2}} .
\end{aligned}
$$

It is not hard to prove that $\left\{-\left(\left(1+\sqrt{1+x^{2}+x}\right) x /(x+1)^{2}\right)\right\}_{x=1}^{\infty}$ is strictly monotone decreasing, and

$$
-1<-\frac{\left(1+\sqrt{1+x^{2}+x}\right) x}{(x+1)^{2}} \leq-\frac{1+\sqrt{3}}{4}=-0.6830127 \ldots
$$

and $\left\{\left(-1+\sqrt{1+x^{2}+x}\right) x /(x+1)^{2}\right\}_{x=1}^{\infty}$ is strictly monotone increasing; we also have

$$
\frac{-1+\sqrt{3}}{4}=0.1830127 \ldots \leq \frac{\left(-1+\sqrt{1+x^{2}+x}\right) x}{(x+1)^{2}}<1 .
$$

Hence, the inequality $I_{x}(\lambda) \leq 0$ always holds for $x \geq 1$ and $\lambda \epsilon$ $[(-1-\sqrt{3}) / 4,(-1+\sqrt{3}) / 4]$. By using $\lim _{x \rightarrow \infty} d^{\prime}(x)=0$, thus $d^{\prime}(x) \geq 0$. It shows that sequence $W_{n}=[1+\lambda /(\lambda+1) n] x_{n}$ is monotone increasing under this condition, and the inequality in this theorem can be proved by using $\lim _{n \rightarrow \infty} W_{n}=e$.

2.2. Further Discussions Based on Introducing the Parameters. Here we study the monotonicity of two new sequences and their relationships with $e$ by introducing real parameter $\lambda$. Two sequences are, respectively, $i_{n}=(1+(\lambda / n))^{n}$ and $j_{n}=(1+(\lambda / n))^{n+1}$, where $\lambda>0$, and it is easy to obtain $\lim _{n \rightarrow \infty} i_{n}=\lim _{n \rightarrow \infty} j_{n}=e^{\lambda}$. Next, we consider their arithmetic mean value sequence, geometric mean value sequence, and harmonic mean value sequence, respectively; consider

$$
\begin{gathered}
A_{1}(n, \lambda)=\frac{i_{n}+j_{n}}{2}=\left(1+\frac{\lambda}{2 n}\right)\left(1+\frac{\lambda}{n}\right)^{n}, \\
G_{1}(n, \lambda)=\left(i_{n} j_{n}\right)^{1 / 2}=\left(1+\frac{\lambda}{n}\right)^{1 / 2}\left(1+\frac{\lambda}{n}\right)^{n}, \\
H_{1}(n, \lambda)=\frac{2 i_{n} j_{n}}{i_{n}+j_{n}}=\left(1+\frac{\lambda}{2 n+\lambda}\right)\left(1+\frac{\lambda}{n}\right)^{n} .
\end{gathered}
$$

Theorem 10. For $0<\lambda \leq 1$ and $n \in N^{*}$, the sequences $A_{1}(n, \lambda)=(1+(\lambda / 2 n))(1+(\lambda / n))^{n}$ are monotone decreasing, and the inequality $e^{\lambda}<(1+(\lambda / 2 n))(1+(\lambda / n))^{n}$ holds.

Proof. We consider $M(x)=x \ln (x+\lambda)-x \ln x+\ln (2 x+\lambda)-$ $\ln (2 x), x>0$; then

$$
\begin{gathered}
M^{\prime}(x)=\ln (x+\lambda)+\frac{x}{x+\lambda}-\ln x-1+\frac{2}{2 x+\lambda}-\frac{1}{x} \\
M^{\prime \prime}(x)=2 \frac{1}{x+\lambda}-\frac{x}{(x+\lambda)^{2}}-\frac{1}{x}-\frac{4}{(2 x+\lambda)^{2}}+\frac{1}{x^{2}} \\
=-\left(\lambda \left(4 x^{3} \lambda+4 x^{2} \lambda^{2}+x \lambda^{3}-4 x^{3}-9 x^{2} \lambda\right.\right. \\
\left.\left.-6 x \lambda^{2}-\lambda^{3}\right)\right) \times\left(x^{2}(x+\lambda)^{2}(2 x+\lambda)^{2}\right)^{-1}
\end{gathered}
$$

Denote the numerator of $M^{\prime \prime}(x)$ in (26) as

$$
\begin{gathered}
N(x):=-\lambda\left(4 x^{3} \lambda+4 x^{2} \lambda^{2}+x \lambda^{3}-4 x^{3}-9 x^{2} \lambda\right. \\
\left.-6 x \lambda^{2}-\lambda^{3}\right):=\lambda Y(x),
\end{gathered}
$$


where $Y(x):=4 x^{3}-4 x^{3} \lambda+9 x^{2} \lambda-4 x^{2} \lambda^{2}+6 x \lambda^{2}-x \lambda^{3}+\lambda^{3}$. Take $\forall p \geq 0$; then $\lambda:=1 /(p+1) \in(0,1]$ and

$$
\begin{aligned}
Y(x):= & 4 x^{3}-\frac{4 x^{3}}{p+1}+\frac{9 x^{2}}{p+1}-\frac{4 x^{2}}{(p+1)^{2}}+\frac{6 x}{(p+1)^{2}} \\
& -\frac{x}{(p+1)^{3}}+\frac{1}{(p+1)^{3}} \\
= & \left(4 x^{3} p^{3}+8 x^{3} p^{2}+4 x^{3} p+9 x^{2} p^{2}\right. \\
& \left.+14 x^{2} p+5 x^{2}+6 x p+5 x+1\right) \times\left((p+1)^{3}\right)^{-1} .
\end{aligned}
$$

It is found that if $0<\lambda \leq 1$ and $x>0$, then $Y(x) \geq 0$. Furthermore, it implies that

$$
N(x)=\lambda Y(x) \geq 0
$$

then $M^{\prime}(x)$ is monotone increasing, and $\lim _{x \rightarrow \infty} M^{\prime}(x)=0$ has been verified, such that $M^{\prime}(x)<0$, and $A_{1}(n, \lambda)=$ $(1+(\lambda / 2 n))(1+(\lambda / n))^{n}$ are monotone decreasing; hence $e^{\lambda}<(1+(\lambda / 2 n))(1+(\lambda / n))^{n}$ can be proved because of $\lim _{n \rightarrow \infty} A_{1}(n, \lambda)=e^{\lambda}$.

Theorem 11. For $0<\lambda \leq 1$ and $n \in N^{*}$, the sequences $G_{1}(n, \lambda)=(1+(\lambda / n))^{1 / 2}(1+(\lambda / n))^{n}$ are monotone decreasing, and the inequality $(1+(\lambda / n))^{1 / 2}(1+(\lambda / n))^{n}>e^{\lambda}$ holds.

Proof. We consider $f_{1}=x \ln (1+(\lambda / x))+(1 / 2) \ln (1+(\lambda / x))$, $x \geq 1$; then

$$
\begin{aligned}
f_{1}^{\prime}(x)= & \ln \left(1+\frac{\lambda}{x}\right)-\frac{\lambda}{x(1+(\lambda / x))}-\frac{\lambda}{2 x^{2}(1+(\lambda / x))} \\
f_{1}^{\prime \prime}(x)= & \frac{-\lambda^{2}}{x^{3}(1+(1 / x))^{2}}+\frac{\lambda}{x^{3}(1+(\lambda / x))} \\
& -\frac{\lambda^{2}}{2 x^{4}(1+(\lambda / x))^{2}}=\frac{-\lambda(2 \lambda x-2 x-\lambda)}{2 x^{2}(x+\lambda)^{2}} \\
= & \frac{(-2 x+1) \lambda^{2}+2 \lambda x}{2 x^{2}(x+\lambda)^{2}} .
\end{aligned}
$$

Denote the numerator of $(31)$ as $\delta(\lambda)=(-2 x+1) \lambda^{2}+2 x \lambda$, which is a quadratic function of $\lambda$. And its discriminant is $\Delta_{1}=4 x^{2}>0$. Then two roots of $\delta(\lambda)$ are

$$
\lambda_{1}=0, \quad \lambda_{2}=\frac{2 x}{2 x-1}
$$

We note that $\{2 x /(2 x-1)\}_{x=1}^{\infty}$ is strict monotone decreasing and $1<(2 x /(2 x-1)) \leq 2, x \geq 1$. According to the characteristic of parabola curve, $\delta(\lambda)=(-2 x+1) \lambda^{2}+2 x \lambda>$ 0 always holds for $0<\lambda \leq 1$ and $x \geq 1$; then from $\lim _{x \rightarrow \infty} f_{1}^{\prime}(x)=0, f_{1}^{\prime}(x) \leq 0$. It shows that the sequences $G_{1}(n, \lambda)$ are monotone decreasing.
Theorem 12. If $\lambda \geq 1$, then $H_{1}(n, \lambda)=\left(2 i_{n} j_{n}\right) /\left(i_{n}+j_{n}\right)=$ $(1+\lambda /(2 n+\lambda))(1+(\lambda / n))^{n}$ are monotone increasing, and the following inequality

$$
\left(1+\frac{\lambda}{2 n+\lambda}\right)\left(1+\frac{\lambda}{n}\right)^{n}<e^{\lambda}
$$

holds for $n \in N^{*}$.

Proof. We consider $\varphi(x)=\ln (2 x+2 \lambda)-\ln (2 x+\lambda)+x \ln (x+$ $\lambda)-x \ln x, x>0$; then

$$
\varphi^{\prime}(x)=\frac{2}{2 x+2 \lambda}-\frac{2}{2 x+\lambda}+\ln (x+\lambda)+\frac{x}{x+\lambda}-\ln x-1,
$$

$$
\begin{aligned}
\varphi^{\prime \prime}(x)= & \frac{-4}{(2 x+2 \lambda)^{2}}+\frac{4}{(2 x+\lambda)^{2}}+\frac{2}{x+\lambda}-\frac{x}{(x+\lambda)^{2}} \\
& -\frac{1}{x} \\
= & -\left(\lambda\left(-4 x^{2}-3 \lambda x+4 \lambda x^{2}+4 x \lambda^{2}+\lambda^{3}\right)\right) \\
& \times\left((x+\lambda)^{2}(2 x+\lambda)^{2} x\right)^{-1} .
\end{aligned}
$$

Denote numerator of (35) as

$$
\gamma(x)=-\lambda\left(-4 x^{2}-3 \lambda x+4 \lambda x^{2}+4 x \lambda^{2}+\lambda^{3}\right):=-\lambda Z(x),
$$

where $Z(x):=-4 x^{2}-3 \lambda x+4 \lambda x^{2}+4 x \lambda^{2}+\lambda^{3}$. Since $\tau+1:=$ $\lambda \geq 1$, that is, $\tau \geq 0$, we have

$$
\begin{aligned}
Z(x):= & -4 x^{2}-3 x(\tau+1)+4 x^{2}(\tau+1) \\
& +4 x(\tau+1)^{2}+(\tau+1)^{3} \\
= & 4 x^{2} \tau+(\tau+1)(4 \tau+1) x+(\tau+1)^{3} \geq 0 .
\end{aligned}
$$

$$
(x>0, \tau \geq 0)
$$

It implies that the inequality $\gamma(x)=-\lambda Z(x) \leq 0$ always holds for $x>0, \lambda \geq 1$. Then $\varphi^{\prime}(x)$ is monotone decreasing, and according to $\lim _{x \rightarrow \infty} \varphi^{\prime}(x)=0$, we have $\varphi^{\prime}(x)>0$. Hence, $H_{1}(n, \lambda)=(1+\lambda /(2 n+\lambda))(1+(\lambda / n))^{n}$ are monotone increasing sequences, and we prove that

$$
e^{\lambda}>\left(1+\frac{\lambda}{2 n+\lambda}\right)\left(1+\frac{\lambda}{n}\right)^{n} .
$$

A remaining issue of Theorem 12 can be described as follows: when $\lambda<1$, are the $H_{1}(n, \lambda)$ monotone increasing or monotone decreasing? With the help of the Bottema (available at the site of http://old.irgoc.org/Soft/ShowSoft. asp?SoftID=15, and the practical implementing Maple codes also are available by the authors' email) (developed by Yang and Xia and based on the Maple programme; see [29] and references therein), we can find that the inequality $\gamma(x) \geq 0$ holds for $0<\lambda \leq(4 / 5), x \geq 1$. Moreover, it can be also shown in Algorithm 1.

Then we can conclude that $H_{1}(n, \lambda), n=1,2, \ldots$, is monotone decreasing under the previous discussion in the proof of the Theorem 12. The further conclusions need to be studied and proven by analytical methods in future work. 
TABLE 1: Using $H_{1}(N, \lambda)$ to approximate the $e^{\lambda}$, when $\lambda=1,3 / 2,2,3,4$ with different $n$.

\begin{tabular}{lccccc}
\hline$n$ & $H_{1}(n, 1)$ & $H_{1}(n, 2)$ & $H_{1}(n, 3)$ & $H_{1}(n, 4)$ & $H_{1}(n, 3 / 2)$ \\
& $e \approx 2.71828$ & $e^{2} \approx 7.3891$ & $e^{3} \approx 20.0855$ & $e^{4} \approx 54.5982$ & 4.480010 \\
\hline 1000 & 2.71828 & 7.3817 & 20.0255 & 54.2724 & 4.481129 \\
3000 & 2.71828 & 7.3866 & 20.0655 & 54.4892 & 4.481353 \\
5000 & 2.71828 & 7.3876 & 20.0735 & 54.5327 & 4.481449 \\
7000 & 2.71828 & 7.3880 & 20.0769 & 54.5514 & 4.481502 \\
9000 & 2.71828 & 7.3882 & 20.0788 & 54.5618 & 4.481536 \\
11000 & 2.71828 & 7.3884 & 20.0801 & 54.5684 & 4.481560 \\
13000 & 2.71828 & 7.3885 & 20.0809 & 54.5730 & 4.481577 \\
15000 & 2.71828 & 7.3886 & 20.0815 & 54.5763 & \\
\hline
\end{tabular}

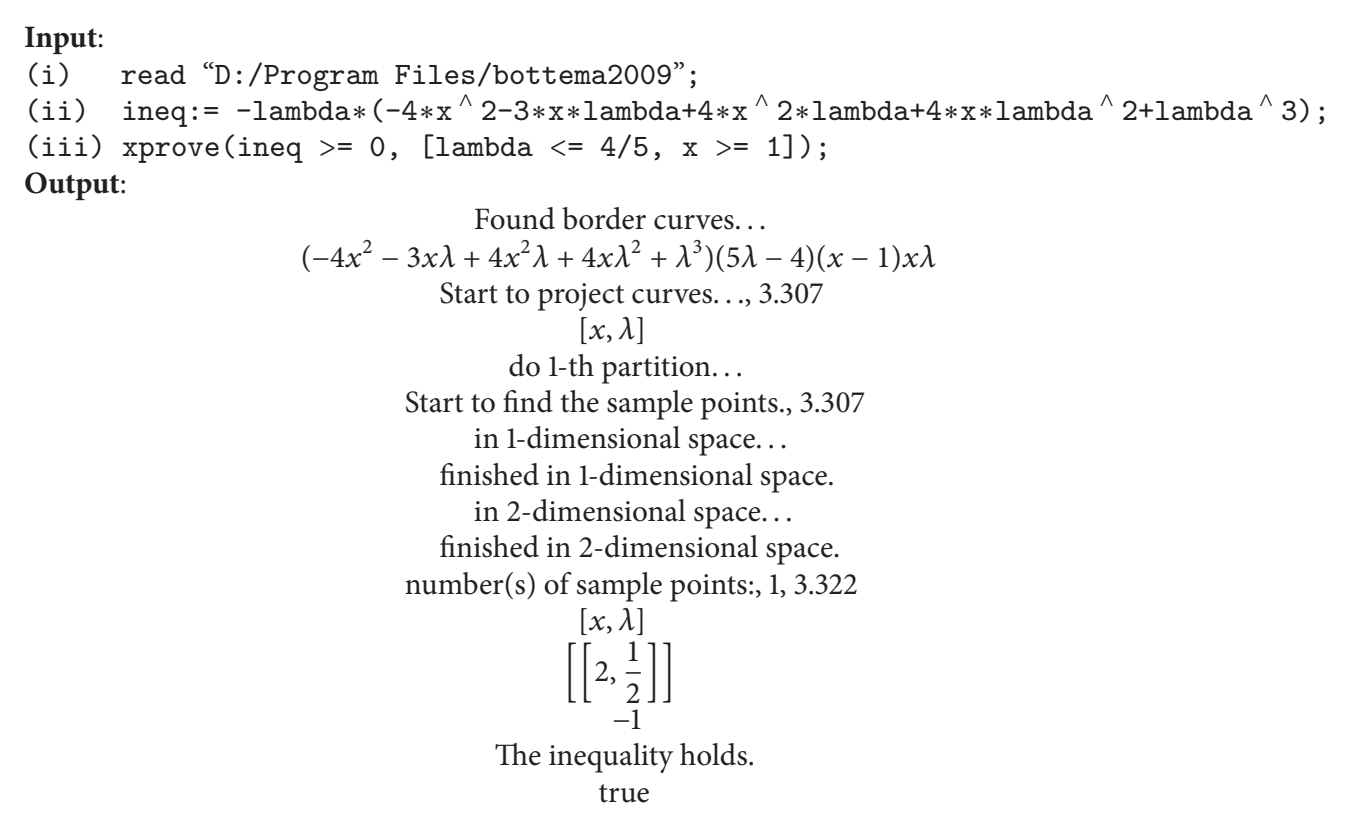

Algorithm 1

\section{Numerical Examples}

In this section, we will display some new upper and lower bounds of the I. Schur inequality by using the Matlab 2011b in a personal computer. We give some figures to show their variation trend; the symbols $L_{0}-L_{4}$ here mean the corresponding real continuous function for $L_{0}=x_{n}(1+$ $(1 / 2 n)), L_{1}=x_{n}(n+1) \ln (1+(1 / n)), L_{2}=\left(x_{n} /(n \ln (1+\right.$ $(1 / n)))), L_{3}=x_{n}\left(1+\left((4 n+1) /\left(8 n^{2}+4 n\right)\right)\right)$, and $L_{4}=$ $x_{n}\left(1+\left((4 n+3) /\left(8 n^{2}+8 n+1\right)\right)\right)$, respectively.

Form Figure 1, we can note that the original upper bound $L_{0}$ of the I. Schur inequality is not better than the other novel upper bounds $L_{1}-L_{4}$; the $L_{1}$ is the best upper bound among five upper bounds. Moreover, we note that the variation curves of $L_{3}, L_{4}$ are greatly similar; they are both the other alternatives. This figure also shows that the improvement of the upper bound of the I. Schur inequality is beneficial.

Firstly, we define the corresponding real continuous function for $T_{1}=x_{n}(1+(1 /(2 n+1))), T_{2}=x_{n}(1+(1 / n))^{1 / 8}$, $T_{3}=x_{n}(1+(1 / n))^{1 / 4}, T_{4}=x_{n}(1+((1 / 100) /(1+(1 / 100)) n))$, and $T_{5}=x_{n}(1+(-1 / 3) /(1-(1 / 3)) n)$, respectively. From Figure 2 , we can note that the original lower bound $T_{1}$ of the I. Schur inequality is better than the other novel lower bounds $T_{2}-T_{5}$; the $T_{3}$ is an alternative among other novel lower bounds. This figure shows that we improve the lower bound of the I. Schur inequality and it is not very successful. But we give some generalized upper and lower bounds for the $e^{\lambda}, \lambda \in(0,1)$; this work is meaningful in terms of the generalized type of the I. Schur inequality.

From Table 1, we find that $H_{1}(n, \lambda)$ can be viewed as a good lower bound of $e^{\lambda}, \lambda \geq 1$ in terms of approximating precision, especially for the case with $\lambda=1$. It is favorable to note that the reliability of the approximation of $e^{\lambda}$ based on $H_{1}(N, \lambda)$ depends on the closeness between $\lambda$ and 1 .

\section{Conclusions}

In the previous sections, we have studied the monotonicity of the sequences and their variants based on various mean 


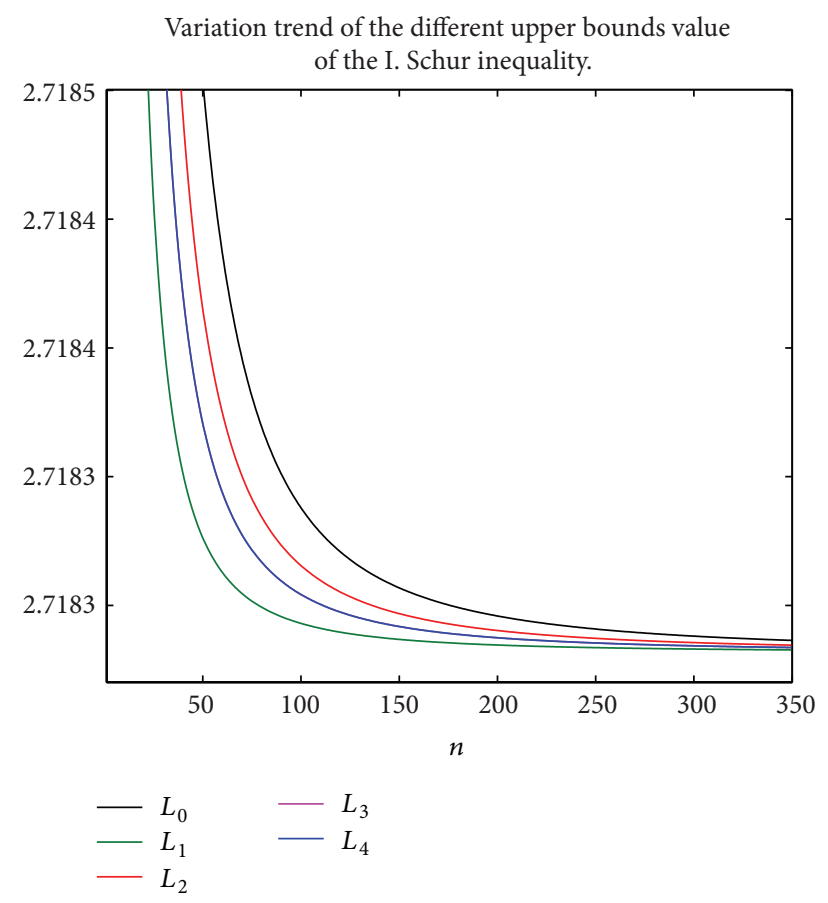

FIGURE 1: The variation trend of the different upper bounds value of the I. Schur inequality.

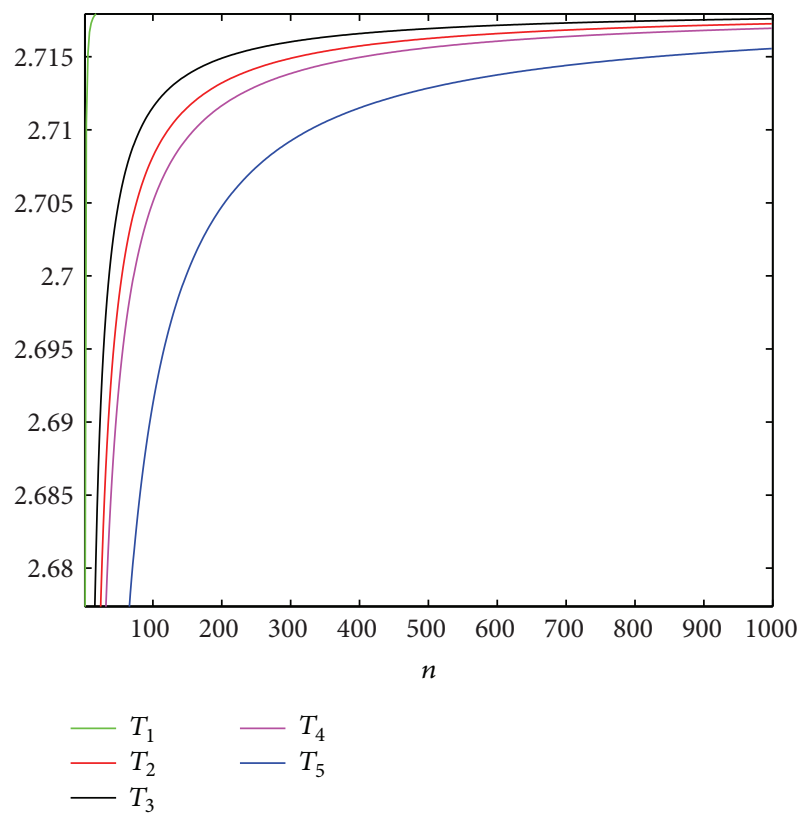

Figure 2: The variation trend of the different lower bounds value of the I. Schur inequality.

values of $x_{n}$ and $y_{n}$, such as the arithmetic mean value, geometric mean value, logarithmic mean value, and harmonic mean value. Besides, we have given some extensions and remarks for known results in the recent literature. At the same time, we extend some ideas and conclusions in [26, 30-32]. In conclusion, it is not hard to find that the conclusions of previous generalized sequences with variable parameters are in terms of the I. Schur type inequality via the parameter techniques and are a kind of extensions of I. Schur type inequality.

\section{Conflict of Interests}

The authors declare that there is no conflict of interests regarding the publication of this paper.

\section{Authors' Contribution}

Xian-Ming Gu carried out the main part of this paper. TingZhu Huang, Wei-Ru Xu, Hou-Biao Li, Liang Li, and Xi-Le Zhao participated in the discussion and corrected the main theorem. All authors read and approved the final paper.

\section{Acknowledgments}

The authors would like to express their thankfulness to the referees for their suggestions while revising this paper. This research is supported by 973 Program (2013CB329404), NSFC (61370147, 61170311, 11101071, and 11301057), the Fundamental Research Funds for the Central Universities (ZYGX2013J106), Chinese Universities Specialized Research Fund for the Doctoral Program (20110185110020), and the Fundamental Research Funds for China Scholarship Council, and the project was sponsored by OATF, UESTC.

\section{References}

[1] J. A. Knox and H. J. Brothers, "Novel series-based approximations to e," The College Mathematics Journal, vol. 30, no. 4, pp. 269-275, 1999.

[2] A. Čižmešija, J. Pečarić, and L. E. Persson, "On strengthened weighted Carleman's inequality," Bulletin of the Australian Mathematical Society, vol. 68, no. 3, pp. 481-490, 2003.

[3] Y. Bicheng, "On Hardy's inequality," Journal of Mathematical Analysis and Applications, vol. 234, no. 2, pp. 717-722, 1999.

[4] X.-J. Yang, Y.-I. Kim, and K.-M. Lo, "Approximation for constant $e$ and its applications," Applied Mathematics and Computation, vol. 206, no. 1, pp. 50-55, 2008.

[5] J.-Z. Liu, X.-D. Zhang, and B. Jiang, "Some generalizations and improvements of discrete Hardy's inequality," Computers and Mathematics with Applications, vol. 63, no. 3, pp. 601-607, 2012.

[6] Z.-X. Lu, Y.-C. Gao, and Y.-X. Wei, "Note on the Carleman's inequality and Hardy's inequality," Computers and Mathematics with Applications, vol. 59, no. 1, pp. 94-97, 2010.

[7] P. Yan and G.-Z. Sun, "A strengthened Carleman's inequality," Journal of Mathematical Analysis and Applications, vol. 240, no. 1, pp. 290-293, 1999.

[8] Z.-T. Xie and Y.-B. Zhong, "A best approximation for constant $e$ and an improvement to Hardy's inequality," Journal of Mathematical Analysis and Applications, vol. 252, no. 2, pp. 994-998, 2000.

[9] C.-P. Chen, W.-S. Cheung, and F. Qi, "Note on weighted Carleman-type inequality," International Journal of Mathematics and Mathematical Sciences, vol. 2005, no. 3, pp. 475-481, 2005. 
[10] B.-Q. Yuan, "Refinements of Carleman's inequality," Journal of Inequalities in Pure and Applied Mathematics, vol. 2, no. 2, pp. 1-10, 2001.

[11] C. Mortici, "Refinements of some bounds related to the constant e," Miskolc Mathematical Notes, vol. 12, no. 1, pp. 105-111, 2011.

[12] X.-J. Yang, “On Carleman's inequality," Journal of Mathematical Analysis and Applications, vol. 253, no. 2, pp. 691-694, 2001.

[13] C.-P. Chen and F. Qi, "On further sharpening of Carleman's inequality," College Mathematics, vol. 21, no. 2, pp. 88-90, 2005 (Chinese).

[14] J.-C. Kuang, Applied Inequalities, Shandong Science and Technology Press, Jinan, China, 4th edition, 2010 (Chinese).

[15] G. Klambauer, Problems and Propositions in Analysis, Lecture Notes in Pure and Applied Mathematics, Marcel Dekker, New York, NY, USA, 1979.

[16] S. K. Khattri, "Three proofs of the inequality $e<(1+1 / n)^{n+0.5}$," American Mathematical Monthly, vol. 117, no. 3, pp. 273-277, 2010.

[17] G. Polya and G. Szego, Problems and Theorems in Analysis I: Series, Integral Calculus, Theory of Functions, Classics in Mathematics, Springer, New York, NY, USA, 1998.

[18] P. Fischer and R. J. Stroeker, "Some interesting logarithmic inequalities and a problem of Pólya and Szegö," Nieuw Archief voor Wiskunde, vol. 12, no. 3, pp. 119-124, 1994.

[19] F. Qi, W. Li, and B.-N. Guo, "Generalizations of a theorem of I. Schur," Applied Mathematics E-Notes, vol. 6, pp. 244-250, 2006.

[20] H.-W. Chen, "On an infinite series for $(1+1 / x) x$ and its application," International Journal of Mathematics and Mathematical Science, vol. 29, no. 11, pp. 675-680, 2002.

[21] H. Alzer and C. Berg, "Some classes of completely monotonic functions," Annales Academiae Scientiarum Fennicae Mathematica, vol. 27, no. 2, pp. 445-460, 2002.

[22] B.-N. Guo and F. Qi, "A property of logarithmically absolutely monotonic functions and the logarithmically complete monotonicity of a power-exponential function," UPB Scientific Bulletin A, vol. 72, no. 2, pp. 21-30, 2010.

[23] X.-Q. Xu and Y.-Q. Yang, "The lower and upper estimations of number e and their lengths," Journal of Jiangxi Normal University (Natural Science), vol. 22, no. 1, pp. 10-11, 1998 (Chinese).

[24] H. Yang and H. Yang, "The arithmetic-geometric mean inequality and the constant $e$," Mathematics Magazine, vol. 74, no. 4, pp. 321-323, 2001.

[25] N. Batir and M. Cancan, "Sharp inequalities involving the

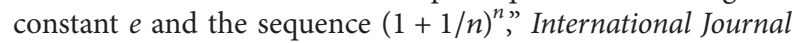
of Mathematical Education in Science and Technology, vol. 40, no. 8, pp. 1101-1109, 2009.

[26] S. Kaijser, L. E. Persson, and A. Öberg, “On Carleman's and Knopp's inequalities," Journal of Approximation Theory, vol. 117, no. 1, pp. 140-151, 2002.

[27] V. A. Zorich, Mathematical Analysis I, Springer, Berlin, Germany, 2004.

[28] H.-G. Zhao and G.-L. Chang, "Two e series of bilateral inequalities," Journal of Zhejiang Normal University (Natural Science), vol. 34, no. 1, pp. 28-32, 2011 (Chinese).

[29] L. Yang and B.-C. Xia, Automated Discovering and Machinery Proving for Inequalities, Science Press, Beijing, China, 2008 (Chinese).

[30] Y. Bicheng and L. Debnath, "Some Inequalities Involving the Constant $e$, and an Application to Carleman's Inequality,"
Journal of Mathematical Analysis and Applications, vol. 223, no. 1, pp. 347-353, 1998.

[31] D.-W. Niu, J. Cao, and F. Qi, "A class of logarithmically completely monotonic functions related to $(1+1 / x)^{x}$ and an application," General Mathematics, vol. 14, no. 4, pp. 97-112, 2006.

[32] J.-L. Li, "Notes on an inequality involving the constant $e$," Journal of Mathematical Analysis and Applications, vol. 250, no. 2, pp. 722-725, 2000. 


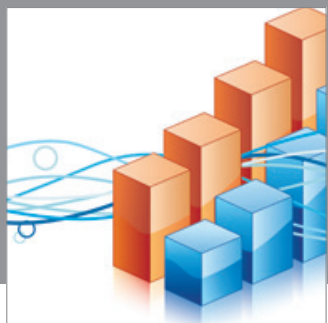

Advances in

Operations Research

mansans

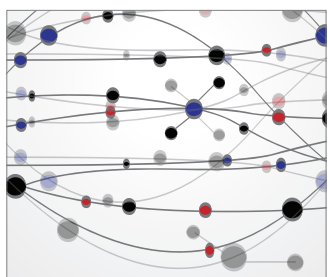

The Scientific World Journal
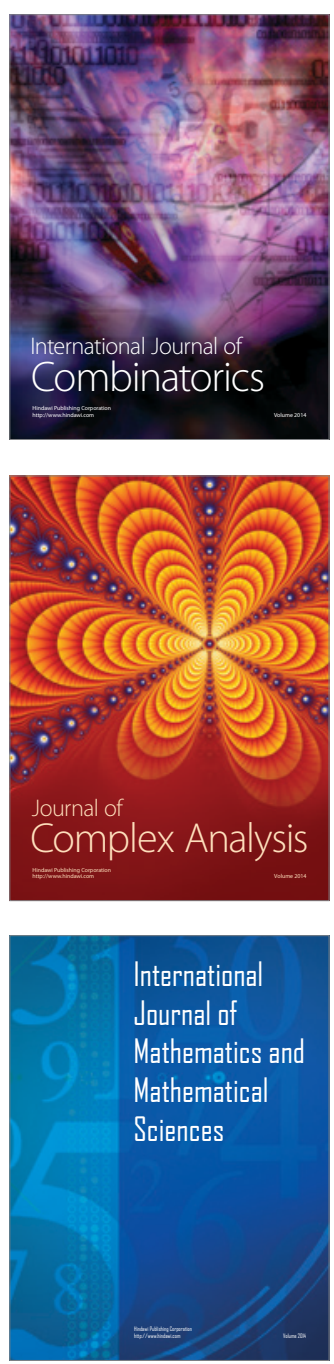
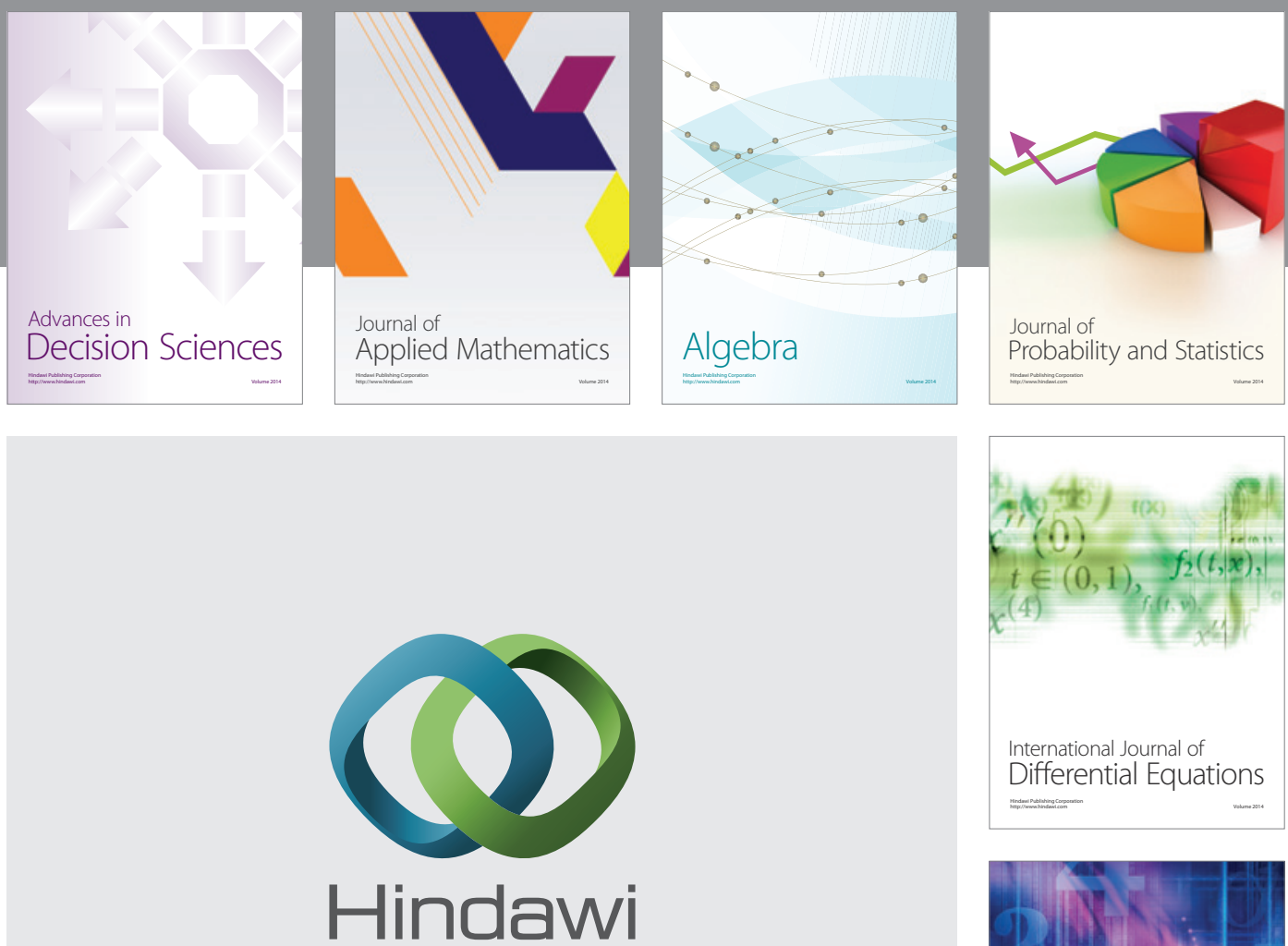

Submit your manuscripts at http://www.hindawi.com
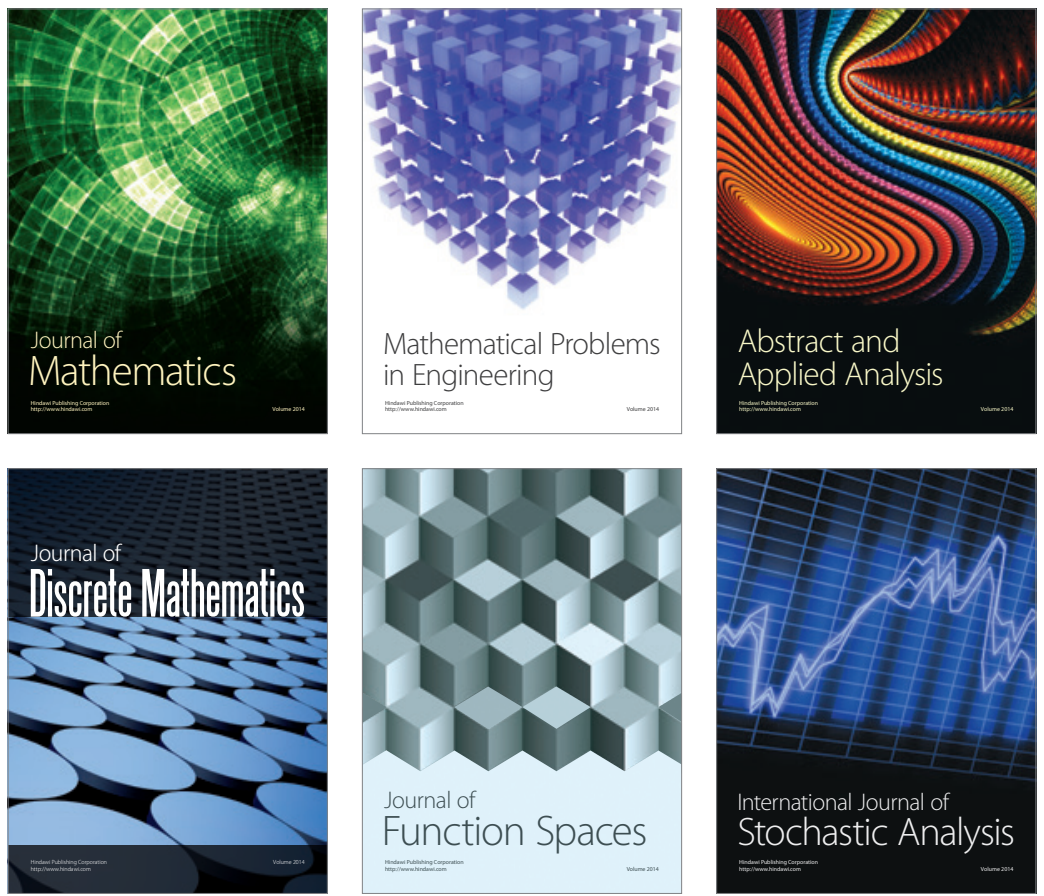

Journal of

Function Spaces

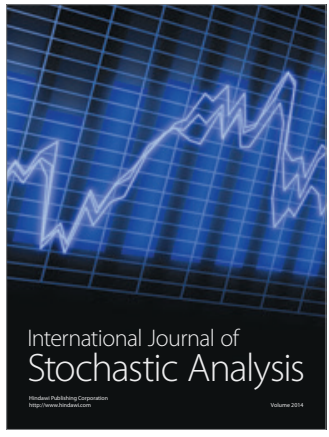

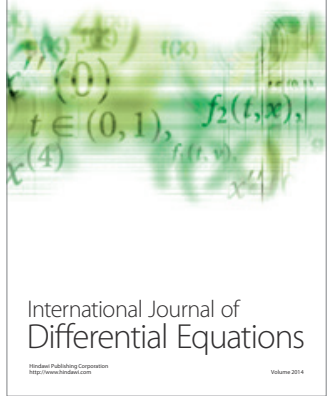
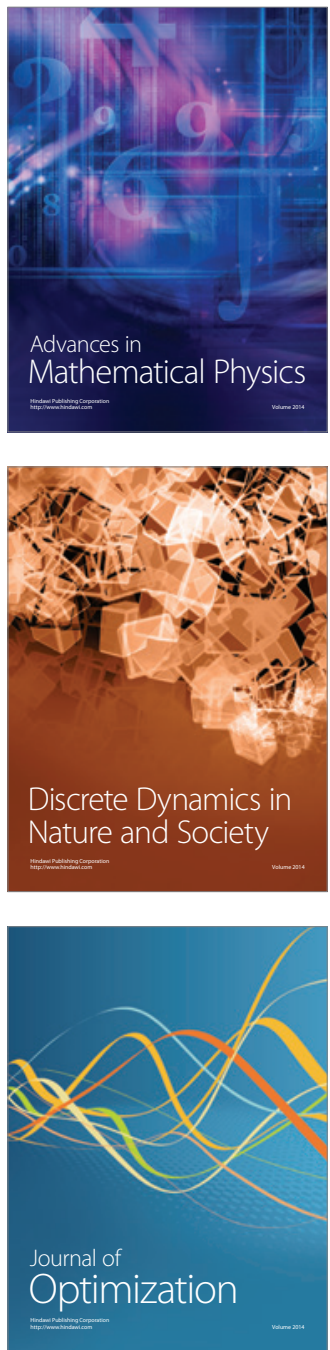